\title{
Research
}

\section{Prediction of risk of death and myocardial infarction in the six months after presentation with acute coronary syndrome: prospective multinational observational study (GRACE)}

\author{
Keith A A Fox, Omar H Dabbous, Robert J Goldberg, Karen S Pieper, Kim A Eagle,
} Frans Van de Werf, Álvaro Avezum, Shaun G Goodman, Marcus D Flather, Frederick A Anderson Jr, Christopher B Granger, for the GRACE Investigators

\begin{abstract}
Objective To develop a clinical risk prediction tool for estimating the cumulative six month risk of death and death or myocardial infarction to facilitate triage and management of patients with acute coronary syndrome.

Design Prospective multinational observational study in which we used multivariable regression to develop a final predictive model, with prospective and external validation.

Setting Ninety four hospitals in 14 countries in Europe, North and South America, Australia, and New Zealand.

Population 43810 patients (21 688 derivation set; 22122 in validation set) presenting with acute coronary syndrome with or without ST segment elevation enrolled in the global registry of acute coronary events (GRACE) study between April 1999 and September 2005.

Main outcome measures Death and myocardial infarction.

Results 1989 patients died in hospital, 1466 died between discharge and six month follow-up, and 2793 sustained a new non-fatal myocardial infarction. Nine factors independently predicted death and the combined end point of death or myocardial infarction in the period from admission to six months after discharge: age, development (or history) of heart failure, peripheral vascular disease, systolic blood pressure, Killip class, initial serum creatinine concentration, elevated initial cardiac markers, cardiac arrest on admission, and ST segment deviation. The simplified model was robust, with prospectively validated C-statistics of 0.81 for predicting death and 0.73 for death or myocardial infarction from admission to six months after discharge. The external applicability of the model was validated in the dataset from GUSTO Ilb (global use of strategies to open occluded coronary arteries).

Conclusions This risk prediction tool uses readily identifiable variables to provide robust prediction of
\end{abstract}

the cumulative six month risk of death or myocardial infarction. It is a rapid and widely applicable method for assessing cardiovascular risk to complement clinical assessment and can guide patient triage and management across the spectrum of patients with acute coronary syndrome.

\section{Introduction}

Patients with acute coronary syndrome present with diverse clinical, electrocardiographic, and enzyme or marker characteristics and experience a wide range of serious cardiovascular outcomes. ${ }^{12}$ Estimated risk, based on clinical characteristics, is challenging and imprecise, yet risk assessment is needed to guide triage and key management decisions.

The large multinational observational global registry of acute coronary events (GRACE) has been used to derive regression models to predict death in hospi$\mathrm{tal}^{3}$ and death after discharge ${ }^{4}$ in patients with acute coronary syndrome. However, a comprehensive risk model is required to predict the cumulative risk of death and death or myocardial infarction during the high risk first six months after initial presentation with acute coronary syndrome, the period when most complications occur. Because triage and management decisions are required within the first hours or days after initial presentation, we derived a risk tool from characteristics of patients with acute coronary syndrome at initial presentation.

\section{Methods}

GRACE methods and design

Full details of the GRACE registry have been published elsewhere. ${ }^{56}$ It was designed to reflect an
This is the abridged version of an article that was posted on bmj.com on 10 October 2006: http://bmj.com/cgi/doi/10.1136/ bmj.38985.646481.55

Full details of inclusion criteria and standard definitions can be found on bmj.com.
Editorial by Junghans and Timmis

Royal Infirmary of Edinburgh,

University of

Edinburgh,

Edinburgh

EH16 4SB

Keith A A Fox

British Heart

Foundation professor

of cardiology

University of

Massachusetts

Medical School,

Worcester, MA

01655, USA

Omar H Dabbous

statistician

Robert J Goldberg

epidemiologist

Frederick A

Anderson Jr

research professor of

surgery

Duke Clinical

Research Institute,

Durham, NC 27705,

USA

Karen S Pieper

statistician

Christopher B

Granger

cardiologist

University of

Michigan

Cardiovascular

Center, Ann Arbor,

MI 48109-0477,

USA

Kim A Eagle

cardiologist

continued over

BMJ 2006;333:1091-4 
Universitair

Ziekenhuis

Gasthuisberg,

Leuven, Belgium

3000

Frans Van de Werf

cardiologist

Dante Pazzanese

Institute of

Cardiology,

04012-909 Sao

Paulo, Brazil

Álvaro Avezum

cardiologist

Terrence Donnelly

Heart Centre,

Division of

Michael's Hospital,

Toronto, ON,

Canada M5B 1W8 cardiologist

Royal Brompton and Harefield NHS

Trust, Royal

London

SW3 6NP

Marcus D Flather

cardiologist

Correspondence to:

K A A Fox

k.a.a.fox@ed.ac.uk
Cardiology, St

Shaun G Goodman

Brompton Hospital,

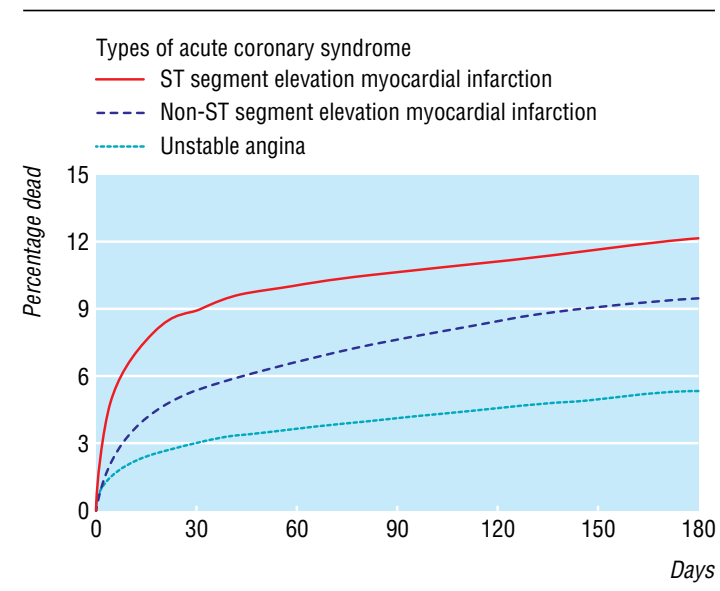

Fig 1 Risk of death from admission to hospital to six months after discharge (patients separated into unstable angina, non-ST segment elevation myocardial infarction, and ST segment elevation myocardial infarction)

unbiased population of patients with acute coronary syndrome in 94 hospitals in 14 countries. All cases were assigned to one of the following categories: ST segment elevation myocardial infarction, non-ST elevation myocardial infarction, or unstable angina (see bmj.com for details).

\section{Statistical methods}

We used two primary end points: all cause death or the composite measure of death or non-fatal myocardial infarction during admission to hospital or after discharge (presentation to six months).

We have summarised the distributions of continuous variables with medians and 25th and 75th centiles and reported the categorical variables as frequencies

Table 1 Final risk models predicting death and death or myocardial infarction from hospital admission to six month follow-up (hazard ratios and 95\% confidence intervals)

\begin{tabular}{|c|c|c|c|c|}
\hline Predictors & $\chi^{2}$ & Death model & $\chi^{2}$ & Death/MI model \\
\hline Age (per 10 year increase) & 505.7 & 1.8 (1.68 to 1.84$)$ & 176.3 & 1.25 (1.21 to 1.29$)$ \\
\hline \multicolumn{5}{|l|}{ Medical history: } \\
\hline Congestive heart failure & 34.2 & $1.5(1.32$ to 1.73$)$ & 22.1 & 1.3 (1.17 to 1.45$)$ \\
\hline Hypertension & 8.8 & $1.2(1.05$ to 1.33$)$ & & - \\
\hline Peripheral vascular disease & 21.8 & 1.4 (1.21 to 1.62$)$ & 10.5 & 1.2 (1.08 to 1.36$)$ \\
\hline $\mathrm{PCl}$ & 8.3 & 0.8 (0.64 to 0.93$)$ & & - \\
\hline \multicolumn{5}{|l|}{ Presentation characteristics: } \\
\hline Pulse (per 30 beats/min increase) & 44.3 & $1.2(1.16$ to 1.31$)$ & & - \\
\hline $\begin{array}{l}\text { Systolic blood pressure (per } 20 \\
\text { mm Hg decrease) }\end{array}$ & 152.0 & $1.2(1.22$ to 1.30$)$ & 52.9 & 1.1 (1.07 to 1.13$)$ \\
\hline Killip class ${ }^{7}$ (per level increase) & 142.8 & 1.5 (1.41 to 1.62$)$ & 126.2 & $1.4(1.30$ to 1.46$)$ \\
\hline $\begin{array}{l}\text { Initial serum creatinine (per } 88 \\
\mu \mathrm{mol} / /^{*} \text { increase) }\end{array}$ & 135.3 & $1.2(1.19$ to 1.29$)$ & 41.1 & 1.1 (1.08 to 1.16$)$ \\
\hline Initial cardiac markers or enzymes & 63.0 & $1.6(1.42$ to 1.78$)$ & 184.3 & 1.7 (1.60 to 1.87$)$ \\
\hline Cardiac arrest & 58.5 & 2.6 (2.00 to 3.32$)$ & 55.4 & 2.2 (1.76 to 2.63$)$ \\
\hline \multicolumn{5}{|l|}{ Findings on electrocardiography: } \\
\hline ST segment deviation & 46.8 & 1.6 (1.41 to 1.88$)$ & & - \\
\hline Left bundle block branch & 10.0 & $1.3(1.10$ to 1.60$)$ & & - \\
\hline $\begin{array}{l}\text { No of leads with ST segment } \\
\text { elevation or depression }\end{array}$ & 20.1 & $1.2(1.10$ to 1.33$)$ & 158.4 & 1.4 (1.34 to 1.49$)$ \\
\hline ST depression, anterior & & - & 36.2 & $1.3(1.22$ to 1.47$)$ \\
\hline ST depression, inferior & & - & 10.8 & 1.2 (1.09 to 1.40$)$ \\
\hline Other changes & & - & 7.2 & 1.1 (1.04 to 1.27$)$ \\
\hline $\begin{array}{l}\text { Hosmer and Lemeshow goodness of } \\
\text { fit test }\end{array}$ & & 0.30 & & 0.42 \\
\hline C-statistic & & 0.82 & & 0.70 \\
\hline
\end{tabular}

$\mathrm{PCl}=$ percutaneous coronary intervention

**Equivalent to $1 \mathrm{mg} / \mathrm{dl}$. and percentages. Events that occurred after six months were censored. See bmj.com for variables included in the analysis from hospital admission to six month follow-up. We used a Cox regression model to compute crude hazard ratios and 95\% confidence intervals to examine the individual relation between each predictor and death and death or myocardial infarction during follow-up (0 to 6 months).

We entered all demographic and clinical variables identified by the crude regression analysis into the stepwise multiple Cox regression (backward) analysis to produce final models for predicting death and death or myocardial infarction. The discriminative power of the final models was assessed by the mean of the area under the receiver operating characteristic (ROC) curve (C-statistic). The model was tested prospectively in a separate dataset in GRACE $(n=22$ 122) and also in an independent external dataset, the GUSTO IIb (global use of strategies to open occluded coronary arteries IIb) dataset, comprising the entire spectrum of patients with acute coronary syndrome (12 142 patients, 4131 with ST elevation myocardial infarction, 8011 with non-ST elevation myocardial infarction) (see bmj.com).

\section{Results}

\section{Study population}

The derivation population comprised 26267 patients with suspected acute coronary syndrome enrolled between 1 April 1999 and 30 September 2002. After exclusions the study population comprised 21688 patients of whom 19931 were alive at six month follow-up.

A total of 1757 (9.1\%) deaths occurred, 1046 in hospital (4.9\% (1046/21 573) among patients with a diagnosis of acute coronary syndrome on admission) and 711 during the period after discharge (4.9\% $(711 / 15265)$. We had no information on mortality (in hospital or after discharge) for 51 patients. In the derivation set, $3110(15.8 \%)$ patients died $(\mathrm{n}=1757)$ or experienced a non-fatal myocardial infarction $(n=1353)$ between presentation and six month follow-up.

Early risks were highest for patients with ST segment elevation myocardial infarction but by six months the risk of death was similar to those with non-ST segment elevation myocardial infarction (fig 1 ). Of those who survived to six months after discharge, $36.2 \%(258 / 711)$ presented with ST segment elevation myocardial infarction compared with 50.0\% (880/ 1757) of those who died during admission or follow-up. Raised cardiac markers were detected in $35.0 \%(6883 / 19688)$ of those who survived compared with $53.2 \%(905 / 1701)$ of those who died.

\section{Validation population}

The validation set comprised 22122 patients enrolled in this multinational registry between 1 October 2003 and 30 September 2005. A total of $1730(9.0 \%)$ patients died between hospital admission and six month follow-up, 948 in hospital (4.3\% among patients with an admission diagnosis of acute coronary syndrome) and 782 (5.4\%) after discharge. No information on mortality was available for 38 patients. In total, 2720 patients died $(n=1730)$ or experienced a non-fatal myocardial infarction $(n=990)$ between presentation and six month follow-up. 


\section{Predictors of mortality}

From admission to six month follow-up, Killip class ${ }^{7}$ and advanced age were the most powerful predictors of death in the univariable analysis.

After multivariable analysis, the highest hazard ratios for death were cardiac arrest on admission and increasing age. These two key prognostic factors were closely followed by raised cardiac markers or enzyme activity and ST segment deviation (table 1).

\section{Risk models predicting death and death or} myocardial infarction

The risk model comprises 14 predictors of death and 12 predictors of death or myocardial infarction. The predictive accuracy of the model was good, with C-statistics of 0.82 for death in hospital and 0.70 for death or myocardial infarction in hospital (table 1). Nine factors independently predicted death and the combined end point in the period from admission to six months after discharge: age, congestive heart failure, peripheral vascular disease, systolic blood pressure, Killip class, initial serum creatinine concentration, positive initial cardiac markers, cardiac arrest on admission, and number of leads with ST deviation. The highest hazard ratio for adverse outcome was for cardiac arrest (table 1).

Prospective and external validation of the GRACE risk score

When we tested the risk model in the prospective validation set, it had excellent predictive accuracy for death and death or myocardial infarction. The predictive accuracy was maintained across the acute coronary syndrome subgroups (table 2).

We validated the model externally using the GUSTO IIb dataset of 12142 patients with acute coronary syndrome. There was excellent discrimination despite the fact that one of the key parameters was not

Table 2 C-statistics for validation of the full model and the simplified model (as used for the nomogram) for all GRACE patients and for acute coronary syndrome subgroups

\begin{tabular}{|c|c|c|c|}
\hline & All patients & STEMI & $\begin{array}{c}\text { Unstable angina/ } \\
\text { NSTEMI }\end{array}$ \\
\hline \multicolumn{4}{|l|}{ All GRACE patients } \\
\hline \multicolumn{4}{|l|}{ Death: } \\
\hline Full model & 0.82 & 0.82 & 0.81 \\
\hline Simplified model & 0.81 & 0.82 & 0.79 \\
\hline \multicolumn{4}{|c|}{ Death or myocardial infarction: } \\
\hline Full model & 0.70 & 0.66 & 0.71 \\
\hline Simplified model & 0.70 & 0.66 & 0.70 \\
\hline \multicolumn{4}{|l|}{ Transferred patients } \\
\hline \multicolumn{4}{|l|}{ Death: } \\
\hline Full model & 0.83 & - & - \\
\hline Simplified model & 0.83 & - & - \\
\hline \multicolumn{4}{|c|}{ Death or myocardial infarction: } \\
\hline Full model & 0.71 & - & - \\
\hline Simplified model & 0.70 & - & - \\
\hline \multicolumn{4}{|l|}{ Model validation* } \\
\hline \multicolumn{4}{|l|}{ Death: } \\
\hline Full model & 0.82 & 0.83 & 0.81 \\
\hline Simplified model & 0.81 & 0.82 & 0.81 \\
\hline \multicolumn{4}{|c|}{ Death or myocardial infarction: } \\
\hline Full model & 0.73 & 0.73 & 0.73 \\
\hline Simplified model & 0.73 & 0.73 & 0.73 \\
\hline
\end{tabular}

STEMI=ST segment elevation myocardial infarction; NSTEMI=non-ST segment elevation myocardial infarction.

*0n subsequent patients with acute coronary syndrome (22 122 enrolled between 1 October 2003 and 30 September 2005).

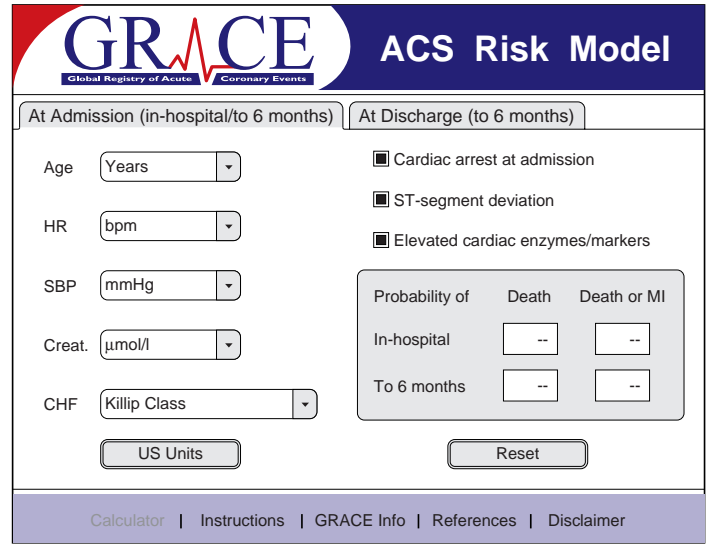

Fig 2 GRACE risk calculator for death or myocardial infarction from admission to hospital to six months after discharge with the simplified model (www.outcomes.org/grace)

recorded in GUSTO IIb (cardiac arrest). The C-statistic for the death model in all patients was 0.82 (C-statistics $=0.80$ for ST segment elevation myocardial infarction and 0.76 for non-ST segment elevation myocardial infarction).

\section{Development of a simplified nomogram for clinical} application

We reduced the overall models to include the most important variables that contained most $(>90 \%)$ of the predictive information. This nomogram retained excellent discriminant characteristics based on eight variables and was used for the calculation of risk (fig 2).

\section{Discussion}

The GRACE risk prediction tool (simplified nomogram) includes variables that are readily available to clinicians even in smaller community hospitals. It provides a novel and widely applicable method of assessing the cumulative six month risk of death and death or myocardial infarction across the spectrum of patients admitted to hospital with acute coronary syndrome.

\section{The need for risk prediction in patients with acute} coronary syndrome

In clinical practice, initial stratification of patients aims to identify those suitable for reperfusion therapy (on the basis of a clinical syndrome and ST segment elevation or other electrocardiographic markers of acute infarction). There is a need for one predictive instrument that performs well in all patients with acute coronary syndrome.

Evidence and practice guidelines suggest that interventional and pharmacological therapies predominantly benefit patients at higher risk. ${ }^{2}{ }^{9}$ Identification of patients at high risk of cardiac ischaemic events, however, remains challenging. ${ }^{10}{ }^{11}$ In addition, the triage of patients into high intensity care units (cardiac care units) is based predominantly on the criteria for reperfusion therapy rather than risk in the patient.

How does the present model differ from previous methods of risk stratification?

Several other multivariable prognostic models have been developed, most of which were derived from 
clinical trial databases or specific subgroups of patients with acute coronary syndrome. Patients with complications and comorbidity tend to be excluded from such trials, thus limiting applicability. Models developed from large claims databases are potentially subject to bias. In contrast, the GRACE registry spans the spectrum of acute coronary syndrome and is based on an unselected contemporary population.

An independent study suggests that the unselected GRACE mortality model is superior to either the TIMI (thrombolysis in myocardial infarction) or the PURSUIT (platelet glycoprotein IIb/IIIa in unstable angina: receptor suppression with eptifibatide) models. ${ }^{12} \mathrm{We}$ have shown that the cumulative ( 0 to six month) GRACE risk model performs well across the spectrum of acute coronary syndrome and has prospective and external validity.

\section{Simplified risk calculation for clinical application}

The simplified model includes most of the predictive information: $>92 \%$ of the total model $\chi^{2}$ for death and $>90 \%$ for death or myocardial infarction (fig 2). The GRACE risk calculator (available at www.outcomes. org/grace) can be used to derive a prognostic score and to estimate the risk of death or the combined risk of death or myocardial infarction in individual patients. This nomogram can be installed into a handheld device or personal computer (data entry takes about 30 seconds) and is also available as a score card. ${ }^{4}$

\section{Limitations}

GRACE is designed to enrol an unselected and generalisable population of patients, though some participating centres are required to obtain informed consent from patients before enrolment. Therefore some patients who died early or who experienced major clinical complications immediately on arrival in hospital may be under-represented. The model may not be appropriate for stratifying low risk patients with non-specific chest pain without acute coronary syndrome, but such patients do not require the same therapeutic and management decisions as those with acute coronary syndrome.

We thank the physicians and nurses who participated in GRACE. The risk calculator is available together with further information about the project and the complete list of participants from www.outcomes.org/grace. We thank Sophie Rushton-Smith for editorial services.

Contributors: See bmj.com.

Funding: The GRACE Registry is supported by an unrestricted educational grant from Sanofi-Aventis to the Center for Outcomes Research, University of Massachusetts Medical School. Sophie Rushton-Smith was funded by Sanofi-Aventis. Competing interests: KAAF has received grant funding from the British Heart Foundation and his department is supported by the British Heart Foundation, Medical Research Council, Wellcome Trust, Sanofi-Aventis, Bristol-Myers Squibb, and MSD. $\mathrm{KAE}$ has received grants from Biosite, Bristol-Myers Squibb, Cardiac Sciences, Blue Cross Blue Shield of Michigan, Hewlett Foundation, Mardigian Fund, Sanofi-Aventis, Varbedian Fund, National Heart, Lung and Blood NIH, and Pfizer. FVdeW has received research grants from Boehringer Ingelheim, SanofiAventis, Proctor and Gamble, Servier, Novartis, MSD, and Schering Plough. ÁA has received funding from Sanofi-Aventis, Population Health Research Institute, and Boehringer Ingelheim. SGG has received funding from AstraZeneca, SanofiAventis, Boehringer Ingelheim, Bristol-Myers Squibb/Sanofi Pharmaceuticals Partnership, Hoffmann-LaRoche Pharmaceuticals, Merck, Novartis, Pfizer, Sanofi-Synthelabo, Schering Corp,

\section{What is already known on this topic}

Specific treatments are indicated in higher or lower risk patients with acute coronary syndrome

Conventional clinical assessment and binary methods for predicting risk based on results of electrocardiography and markers of injury are not sufficiently accurate

Previous risk models were based on subgroups of patients with acute coronary syndrome and were derived from large clinical trials or healthcare claims databases

\section{What this study adds}

The GRACE risk tool can be used to predict the cumulative risk of death and death or myocardial infarction in the period from admission to hospital to six months after discharge

The tool is simple to apply, robust, externally validated, and applicable to patients across the complete spectrum of acute coronary syndrome

and Millennium Pharmaceuticals. MDF, FAA, CBG, and BK have all received funding from Sanofi-Aventis.

Ethical approval: Approval was obtained from local institutional review boards.

1 Van de Werf F, Ardissino D, Betriu A, Cokkinos DV, Falk E, Fox KA, et al. Management of acute myocardial infarction in patients presenting with ST-segment elevation. The task force on the management of acute myocardial infarction of the European Society of Cardiology. Eur Heart J 2003;24:28-66.

2 Braunwald E, Antman EM, Beasley JW, Califf RM, Cheitlin MD, Hochman JS, et al. ACC/AHA guidelines for the management of patients with unstable angina and non-ST-segment elevation myocardial infarction. $J$ Am Coll Cardiol 2000;36:970-1062.

3 Granger CB, Goldberg RJ, Dabbous OM, Pieper KS, Eagle KA, Goodman SG, et al. Predictors of hospital mortality in the global registry of acute coronary events. Arch Int Med 2003;163:2345-53.

4 Eagle KA, Lim MJ, Dabbous OH, Pieper KS, Goldberg RJ, Van de Werf F, et al. A validated prediction model for all forms of acute coronary et al. A validated prediction model for all forms of acute coronary
syndrome: estimating the risk of 6 -month postdischarge death in an syndrome: estimating the risk of 6-month po
international registry. JAMA 2004;291:2727-33.

5 GRACE Investigators. Rationale and design of the GRACE (global registry of acute coronary events) project: a multinational registry of patients hospitalized with acute coronary syndrome. Am Heart J 2001;141:190-9.

6 Eagle KA, Goodman SG, Avezum A, Budaj A, Sullivan CM, Lopez-Sendon J. Practice variation and missed opportunities for reperfusion in ST-segment-elevation myocardial infarction: findings from the global registry of acute coronary events (GRACE). Lancet 2002;359:373-7.

7 Killip T 3rd, Kimball JT. Treatment of myocardial infarction in a coronary care unit. A two year experience with 250 patients. Am J Cardiol 1967;20:457-64.

8 Bertrand ME, Simoons ML, Fox KA, Wallentin LC, Hamm CW, McFadden E, et al. Management of acute coronary syndrome: acute coronary syndrome without persistent ST segment elevation; recommendations of the task force of the European Society of Cardiology. Eur Hear J 2000;21:1406-32.

9 Guideline for the management of patients with acute coronary syndrome without persistent ECG ST segment elevation. British Cardiac Society Guidelines and Medical Practice Committee and Royal College of Physicians Clinical Effectiveness and Evaluation Unit. Heart 2001;85:133-42.

10 Grover SA, Lowensteyn I, Esrey KL, Steinert Y, Joseph L, Abrahamowicz M. Do doctors accurately assess coronary risk in their patients? Preliminary results of the coronary health assessment study. BMJ
1995;310:975-8.

11 McManus RJ, Mant J, Meulendijks CF, Salter RA, Pattison HM, Roalfe AK, et al. Comparison of estimates and calculations of risk of coronary heart disease by doctors and nurses using different calculation tools in general practice: cross sectional study. BMJ 2002;324:459-64.

12 De Araujo Goncalves P, Ferreira J, Aguiar C, Seabra-Gomes R. TIMI PURSUIT, and GRACE risk scores: sustained prognostic value and interaction with revascularization in NSTE-ACS. Eur Heart J 2005;26:865-72.

(Accepted 12 September 2006)

doi 10.1136/bmj.38985.646481.55 